\title{
A comparison of cardiovascular magnetic resonance and single photon emission computed tomography (SPECT) perfusion imaging in left main stem or equivalent coronary artery disease: a CE-MARC substudy
}

James R. J. Foley ${ }^{1}$, Ananth Kidambi ${ }^{1}$, John D. Biglands', Neil Maredia ${ }^{1}$, Catherine J. Dickinson ${ }^{2}$, Sven Plein ${ }^{1}$ and John P. Greenwood ${ }^{1 *}$

\begin{abstract}
Background: Assessment of left main stem (LMS) stenosis has prognostic and therapeutic implications. Data on assessment of LMS disease by cardiovascular magnetic resonance (CMR) and single photon emission computed tomography (SPECT) are limited. CE-MARC is the largest prospective comparison of CMR and SPECT against quantitative invasive coronary angiography (QCA) for detection of coronary artery disease (CAD), and provided the framework for this evaluation. The aims of this study were to compare diagnostic accuracy of visual and quantitative perfusion CMR to SPECT in patients with LMS stable CAD.

Methods: Fifty-four patients from the CE-MARC study were included: 27 (4\%) with significant LMS or LMS-equivalent disease on QCA, and 27 age/sex-matched patients with no flow-limiting CAD. All patients underwent multi-parametric CMR, SPECT and QCA. Performance of visual and quantitative perfusion CMR by Fermi-constrained deconvolution to detect LMS disease was compared with SPECT.
\end{abstract}

Results: Of 27 patients in the LMS group, 22 (81\%) had abnormal CMR and 16 (59\%) had abnormal SPECT. All patients with abnormal CMR had abnormal perfusion by visual analysis. CMR demonstrated significantly higher area under the curve (AUC) for detection of disease $(0.95 ; 0.85-0.99)$ over SPECT $(0.63 ; 0.49-0.76)(p=0.0001)$. Global mean stress myocardial blood flow (MBF) by CMR in LMS patients was significantly lower than controls $(1.77 \pm 0.72 \mathrm{ml} / \mathrm{g} / \mathrm{min}$ vs. $3.28 \pm 1.20 \mathrm{ml} / \mathrm{g} / \mathrm{min}, p<0.001)$. MBF of $<2.08 \mathrm{ml} / \mathrm{g} / \mathrm{min}$ had sensitivity of $78 \%$ and specificity of $85 \%$ for diagnosis of LMS disease, with an AUC $(0.87 ; 0.75-0.94)$ not significantly different to visual CMR analysis $(p=0.18)$, and more accurate than SPECT $(p=0.003)$.

Conclusion: Visual stress perfusion CMR had higher diagnostic accuracy than SPECT to detect LMS disease. Quantitative perfusion CMR had similar performance to visual CMR perfusion analysis.

Keywords: Coronary artery disease, Left main stem disease, Cardiovascular magnetic resonance, SPECT, Quantitative perfusion

\footnotetext{
* Correspondence: j.greenwood@leeds.ac.uk

${ }^{1}$ Multidisciplinary Cardiovascular Research Centre \& The Division of

Biomedical Imaging, Leeds Institute of Cardiovascular and Metabolic

Medicine, University of Leeds, Leeds LS2 9JT, UK

Full list of author information is available at the end of the article
} 


\section{Background}

Left main stem (LMS) coronary artery disease (CAD) is found in approximately $5 \%$ of patients with stable angina and in approximately $7 \%$ of patients presenting with an acute myocardial infarction [1]. Significant LMS disease is typically defined as a stenosis of $\geq 50 \%$ and LMS equivalent as $\geq 70 \%$ stenosis of both the proximal left anterior descending artery (LAD) and proximal circumflex artery (LCx). Significant LMS disease is associated with poor clinical outcomes, with an untreated 3-year survival of $50 \%$ in those with $>50 \%$ stenosis dropping to $41 \%$ in those with stenosis $>70 \%[2,3]$. Several studies have demonstrated survival benefit for revascularisation of significant LMS stenosis $[4,5]$. Thus, accurate detection and functional assessment of the degree of LMS stenosis has both important prognostic and therapeutic implications.

Patients evaluated for suspected CAD frequently undergo functional imaging, which may include single-photon emission computed tomography (SPECT) or cardiovascular magnetic resonance (CMR) imaging. A normal myocardial perfusion study by either of these techniques is associated with an excellent long-term prognosis [6-8]. Published data on the utility of SPECT for the diagnosis of LMS disease are limited, with variable diagnostic accuracy reported [9-12]. Equally, the diagnostic accuracy of stress perfusion CMR is poorly established in LMS disease.

The Clinical Evaluation of MAgnetic Resonance imaging in Coronary heart disease (CE-MARC) study [13, 14] was a large prospective study of patients with suspected CAD; 752 patients were enrolled and all were scheduled to undergo CMR, SPECT and the reference standard invasive coronary angiography. Using the CE-MARC dataset, we hypothesised that CMR would have a greater diagnostic accuracy than SPECT for the detection of LMS or LMS equivalent $\mathrm{CAD}$, and that quantitative CMR perfusion analysis would improve diagnostic discrimination compared to visual analysis.

\section{Methods}

\section{Subjects}

All patients with LMS disease $\geq 50 \%$, and left main equivalent ( $\geq 70 \%$ stenosis of proximal LAD and LCx arteries) by quantitative coronary angiography (QCA) were selected from the CE-MARC population, together with an equal number of control patients without significant stenosis on $\mathrm{X}$-ray angiography. The control patients were independently matched to the LMS group for age, sex and cardiovascular risk factors. The inclusion criteria and full imaging protocol for CE-MARC have been previously reported [14]. In brief, inclusion criteria were: stable chest pain thought to be angina pectoris, at least one cardiovascular disease risk factor, suitability for coronary revascularisation if required and in sinus rhythm. Exclusion criteria were: previous coronary artery bypass surgery, evidence of crescendo angina or acute coronary syndrome, contraindication to CMR imaging or adenosine infusion, and chronic renal failure. The study was performed in accordance with the Declaration of Helsinki (October 2000), with all patients providing informed written consent. The study protocol and other relevant documentation had been approved by the National Research Ethics Committee.

\section{CMR protocol}

Patients underwent perfusion-CMR on a $1.5 \mathrm{~T}$ scanner (Philips Medical Systems, Best, The Netherlands) equipped with "Master" gradients $(30 \mathrm{mT} / \mathrm{m}$ peak gradient, $150 \mathrm{mT} /$ $\mathrm{m} / \mathrm{ms}$ slew rate) and a five-element cardiac phased-array receiver coil. Stress perfusion imaging was performed using intravenous adenosine $(140 \mathrm{mcg} / \mathrm{kg} / \mathrm{min})$ infused for $4 \mathrm{~min}$. Perfusion imaging was performed every heartbeat during the first-pass in 3 short-axis imaging planes, representing the basal, midventricular, and apical myocardial segments. Images were acquired by using a T1-weighted saturation recovery turbo field-echo imaging sequence, using a shared (non-slice-selective) saturation pulse. A bolus of $0.05 \mathrm{mmol} / \mathrm{kg}$ gadopentetate dimeglumine [Gd-DTPA], (Magnevist, Bayer Schering Health Care Limited, UK) followed by a $15 \mathrm{ml}$ saline flush was administered at $5 \mathrm{ml} / \mathrm{s}$ into an antecubital vein by a power injector (Medrad Spectris Solaris, Medrad Inc., Warrendale, Pennsylvania, USA). Resting myocardial perfusion was then assessed and the data obtained with identical parameters as for the resting perfusion acquisition. The CMR protocol also included cine imaging for assessment of left ventricular (LV) function and late gadolinium enhancement imaging (LGE) [14].

\section{SPECT protocol}

SPECT radionuclide imaging was carried out on a dedicated cardiac gamma camera (MEDISO Cardio-C, Budapest, Hungary), using a two-day scanning protocol, the radioisotope tracer ${ }^{99 \mathrm{~m}}$ Tc tetrofosmin (Myoview), with a standard dose of $400 \mathrm{MBq}$, weight-adjusted to a maximum of $600 \mathrm{MBq}$, per examination. Stress and rest ECG-gated SPECT images were acquired. The stress imaging protocol was performed using intravenous adenosine $(140 \mathrm{mcg} / \mathrm{kg} /$ $\mathrm{min}$ ) for $4 \mathrm{~min}$ followed by isotope injection to minimise variation between SPECT and CMR [14].

\section{X-ray angiography}

All patients underwent invasive X-ray coronary angiography by a cardiologist (blinded to SPECT and CMR results).

\section{CMR analysis}

The methods for the visual analysis of CMR in CE-MARC have been described previously [14]. As per the original analysis, CMR was deemed positive if one or more abnormality of perfusion, wall motion abnormality or scar was present $[13,14]$. 
For quantitative perfusion analysis, perfusion CMR data were exported in DICOM format and post-processed offline using the software cvi42, (version 5.1.0, Circle Cardiovascular Imaging, Calgary, Alberta, Cananda.) Contours depicting the myocardium and a region within the LV blood pool were drawn manually (Fig. 1). These contours were copied to all time frames and manually adjusted for breathing motion by using rigid translation. The myocardium was subdivided into six circumferentially equidistant regions in the basal and middle sections and four in the apical section according to the standard American Heart Association (AHA) model [15].

Quantitative perfusion parameters were calculated using in-house software written in Matlab (Mathworks, Natick, Massachusetts, USA) [16]. Myocardial blood flow (MBF) was estimated using Fermi-constrained deconvolution [17]. Blood pool and myocardial curves were converted to contrast agent concentrations assuming a linear relationship between signal intensity and concentration as previously described [16]. An assumed native blood $\mathrm{T}_{1}$ value of $1435 \mathrm{~ms}$ and a contrast agent relaxivity of $4.3 \mathrm{~s}^{-1} \cdot \mathrm{mM}^{-1}$ was used. The arterial input function was taken from the basal slice (which had the shortest preparation delay). Concentration curves were baseline subtracted, corrected for temporal shifts between the arterial input function and the myocardial curves and limited to the first pass of contrast through the left ventricle using previously described automated methods $[16,18]$. Myocardial perfusion reserve (MPR) was calculated as the ratio of stress MBF to rest MBF. Segmental MBF and MPR were averaged to produce per-patient indices for statistical analysis. This was performed with 16 segments to give a global myocardial value, and separately for segments in the LMS territory. The LMS territory comprised segments 1, 2, 5-8, 11-14 and 16 [15]. A quantitative SSS was produced by applying the optimal MBF value derived by Youden's index (as detailed in the statistical methods) to the MBF generated in each of the 16 segments for each patient.

\section{X-ray angiography analysis}

X-ray angiography images were analysed by two cardiologists experienced in invasive coronary angiography. QCA analysis was performed off-line using QCAPlus software (Sanders Data Systems, Palo Alto, California, USA). For all LMS patients, visual and quantitative analysis of the invasive angiogram were concordant.

\section{SPECT analysis}

SPECT data sets were analysed in a blinded manner, simultaneously by a cardiologist with $>10$ years' experience in nuclear cardiology and an experienced medical physicist. Evidence of ischaemia by visual comparison of rest/stress perfusion scans, based on the standard 17segment AHA model, was performed. Additionally, evidence of ischaemia by semi-quantitative scoring (using the QPS 20 segment) (QPS, Cedars-Sinai Medical Center, Los Angeles, California, USA) was also performed. Nonperfusion markers of significant $C A D$, such as transient LV dilatation (TID) and increased right ventricular uptake were also taken in to consideration as felt appropriate by the reporting team.

\section{Data analysis and statistics}

Statistical analysis was performed using commercially available software (SPSS, version 22.0, International Business Machines, Armonk, New York, USA). Two-sided $p$ values $\leq 0.05$ were considered to be statistically significant. Data were compared using Student's $t$-test for continuous

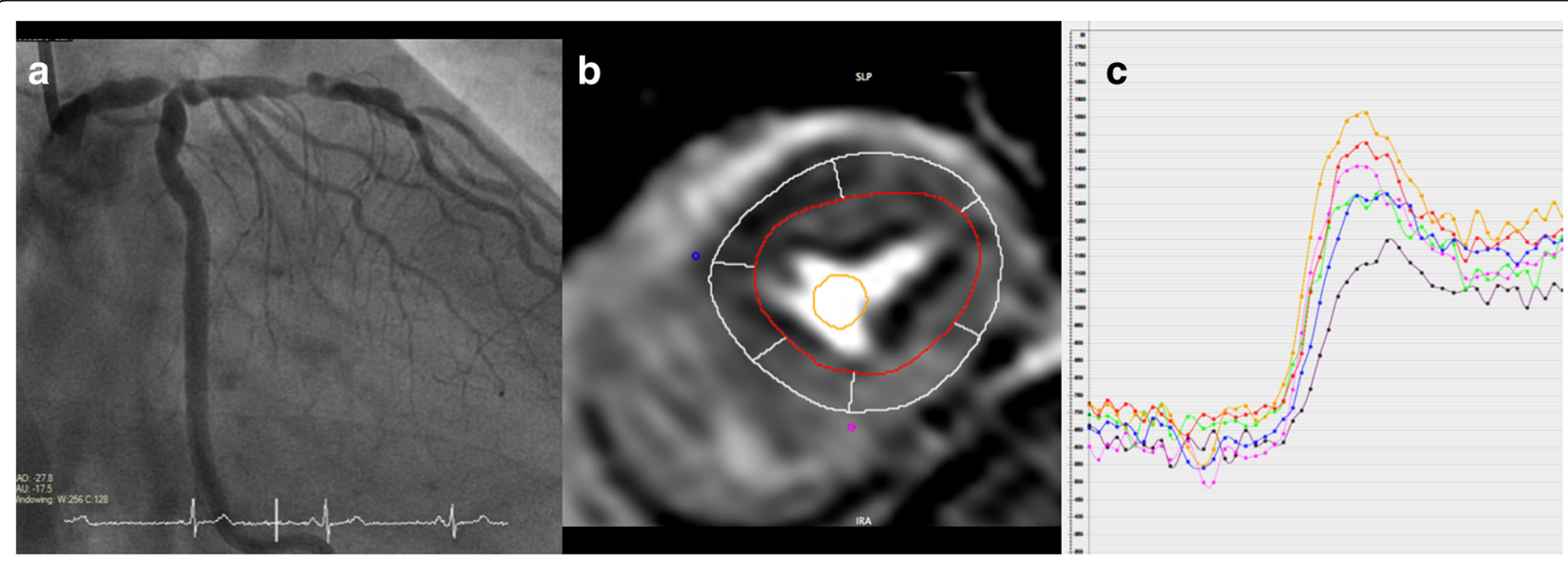

Fig. 1 Image panel showing angiography and CMR perfusion of patient with left main stem (LMS) disease. Panel a shows angiography with a critical distal LMS lesion. The corresponding mid-slice CMR stress perfusion (b) demonstrates a perfusion defect in septum, anterior and lateral wall. Myocardial curves (c) of the same mid ventricular slice demonstrates hypoperfusion in the segments subtended by the LMS. Orange and red lines represent the inferior and infero-lateral segments respectively and show higher signal intensity corresponding with no hypoperfusion in these segments 
variables and Fisher's Exact test for proportions, independent samples $t$ tests and Pearson's correlation coefficients as necessary. Normality for MBF values in the normal comparison group was evaluated using a Q-Q plot and Shapiro-Wilk test. Receiver operating characteristic (ROC) curve analysis for diagnostic tests were compared using the method described by DeLong et al. [19]. For quantitative perfusion analysis, the optimal sensitivity and specificity of quantitative parameters were derived by calculating Youden's index [20]. The sensitivity and specificity and ROC analysis were based on the 54 patients.

\section{Results}

\section{Visual analysis}

Twenty-seven (4\%) patients of the 729 patients that received invasive angiography from CE-MARC were identified to have LMS or LMS equivalent disease by invasive angiography. Twenty-two patients had true LMS disease and 5 patients had LMS equivalent disease. Patient characteristics are shown in Table 1.

All patients had completed CMR, SPECT and angiography studies. Detection rates for CAD by both CMR and SPECT are shown in Table 2. Multi-parametric CMR detected evidence of CAD in a non-significantly higher proportion of patients with LMS disease than SPECT $(81 \%$ vs. $59 \%, p=0.14)$. All patients with abnormal multi-parametric CMR also had abnormal perfusion CMR by visual analysis. One patient was deemed a false negative by SPECT that had 1 segment of inferior ischaemia. For CMR, the average SSS for LMS patients

Table 1 Patient characteristics

\begin{tabular}{llll}
\hline Patient characteristic & LMS & Controls & $P$ \\
\hline $\mathrm{N}$ & 27 & 27 & \\
Age (years) & $65 \pm 7$ & $64 \pm 6$ & 0.45 \\
Male & $23(85 \%)$ & $23(85 \%)$ & 1.0 \\
Body mass index $\left(\mathrm{kg} / \mathrm{m}^{2}\right)$ & $27.5 \pm 3.89$ & $27.0 \pm 2.87$ & 0.60 \\
Current smoker & $5(19 \%)$ & $4(15 \%)$ & 1.0 \\
Blood pressure $(\mathrm{mmHg})$ & $134 / 74 \pm 20 / 10$ & $140 / 76 \pm 19 / 7$ & $0.27 / 0.43$ \\
Hypertension & $12(44 \%)$ & $17(62 \%)$ & 0.27 \\
Total cholesterol (mmol/L) & $5.3 \pm 1.4$ & $4.8 \pm 1.2$ & 0.25 \\
Diabetes mellitus & $5(19 \%)$ & $5(19 \%)$ & 1.0 \\
Family history of CAD & $14(52 \%)$ & $13(48 \%)$ & 1.0 \\
Significant CAD & & & \\
- LMS & $22(81 \%)$ & $0(0 \%)$ & $<0.001$ \\
- LAD & $17(63 \%)$ & $0(0 \%)$ & $<0.001$ \\
- LCX & $11(41 \%)$ & $0(0 \%)$ & $<0.001$ \\
- RCA & $11(41 \%)$ & $0(0 \%)$ & $<0.001$ \\
\hline
\end{tabular}

Data as mean \pm SD or $\mathrm{n}(\%)$

${ }^{a} C A D$ coronary artery disease, $L A D$ left anterior descending coronary artery, $L C X$ left circumflex coronary artery, $R C A$ right coronary artery
Table 2 Imaging findings

\begin{tabular}{|c|c|c|c|}
\hline Imaging finding & LMS & Control & $P$ \\
\hline \multicolumn{4}{|l|}{ CMR } \\
\hline - RWMA $A^{a}$ positive & 17 (63\%) & $0(0 \%)$ & $<0.001$ \\
\hline - FPP positive & 22 (81\%) & $1(4 \%)$ & $<0.001$ \\
\hline - LGE positive & 15 (56\%) & $0(0 \%)$ & $<0.001$ \\
\hline - Overall positive & 22 (81\%) & $1(4 \%)$ & $<0.001$ \\
\hline \multicolumn{4}{|l|}{ SPECT } \\
\hline - RWMA positive & 10 (37\%) & $6(22 \%)$ & 0.37 \\
\hline - Fixed defect & $6(22 \%)$ & $5(19 \%)$ & 1.0 \\
\hline - Inducible defect & 17 (63\%) & $4(15 \%)$ & $<0.001$ \\
\hline$-\mathrm{TID}$ & $1(4 \%)$ & $1(4 \%)$ & 1.0 \\
\hline - RV uptake & 17 (63\%) & 14 (52\%) & 0.58 \\
\hline - Overall positive & 16 (59\%) & $3(11 \%)$ & $<0.001$ \\
\hline
\end{tabular}

was $13.0 \pm 9.5$, and for controls $0.67 \pm 1.0(p<0.001)$. For SPECT, the average SSS for LMS patients was $5.15 \pm 6.5$, and for controls $1.93 \pm 2.3(p=0.02)$. ROC analysis demonstrated a significantly higher area under the curve (AUC) for detection of LMS disease by visual CMR analysis compared to SPECT (0.95 vs. 0.63; $p=0.0001$, Fig. 2).

\section{Quantitative CMR perfusion analysis}

Table 3 shows the results of the quantitative CMR perfusion analysis. Mean stress MBF and mean MPR were both significantly lower in LMS patients compared to controls $(p<0.001)$; resting MBF was similar between the LMS and control groups $(p=0.14)$.

ROC analysis (Fig. 3) demonstrated the highest AUC (0.88) for global MBF as an association with LMS disease. Global MBF of $<2.08 \mathrm{ml} / \mathrm{g} / \mathrm{min}$ was associated with a sensitivity of $78 \%$ and specificity of $85 \%$ for diagnosis of significant LMS disease. A quantitative SSS was produced using this value; this score had an AUC not significantly different to CMR visual analysis $(p=0.18)$, and more accurate than SPECT ( $p=0.003$, Fig. 4 ).

Table 4 shows sensitivity, specificity and predictive values for overall visual analysis by multi-parametric CMR and SPECT, and quantitative analysis by CMR global MBF.

\section{Discussion}

This exploratory analysis of the CE-MARC study has demonstrated the diagnostic accuracy of CMR and SPECT in the setting of LMS (or equivalent) CAD. The main finding is that in patients with stable suspected CAD, CMR firstpass perfusion imaging as part of a multi-parametric protocol more accurately detected evidence of CAD in LMS patients than SPECT. Additionally, quantitative CMR 


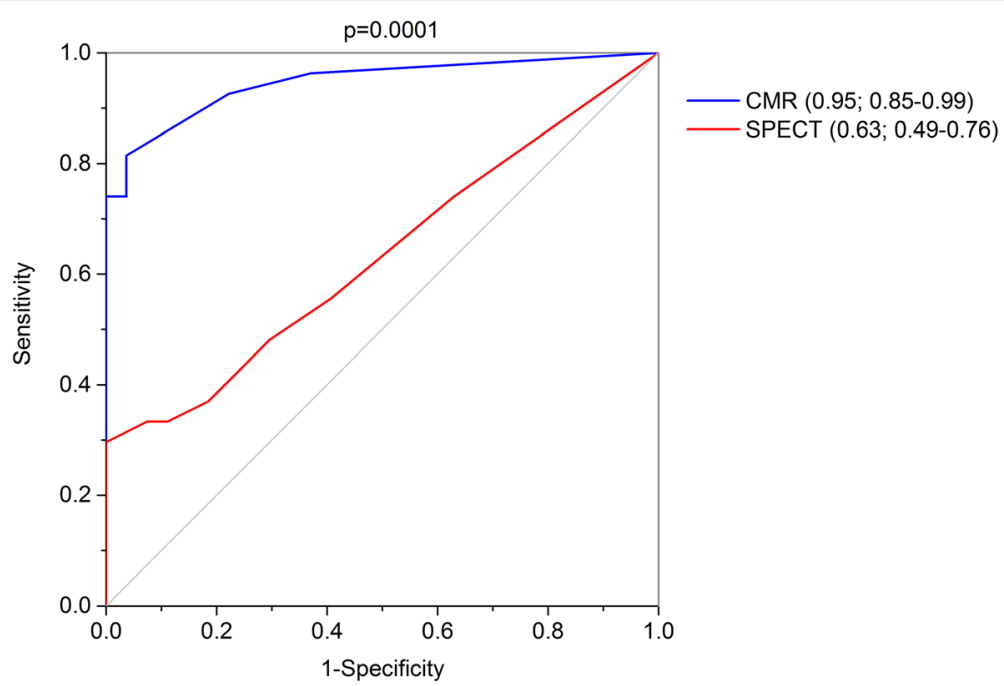

Fig. 2 ROC curves for visual summed stress scores for CMR and single photon emission computed tomography (SPECT). Numbers in parentheses indicates area under the curve (AUC) with 95\% confidence intervals

perfusion showed high diagnostic accuracy for the detection of LMS disease with global MBF as the most diagnostic, however quantitative perfusion did not outperform visual CMR perfusion analysis.

CMR is established as a cost effective investigation with high diagnostic accuracy compared to SPECT for the diagnosis of suspected CAD [13, 21-23]. Previous data on the diagnostic accuracy of SPECT and CMR in LMS disease are sparse. Thus far there are no studies specifically investigating the diagnostic accuracy of CMR for LMS disease. The MR-IMPACT study [24], a multicentre comparison of CMR and SPECT in 234 patients, included eight patients with LMS disease, while MRIMPACT II analysed 465 patients of which 14 had LMS disease [22]; in neither of these studies were patients with LMS disease separately analysed. The majority of studies validating CMR perfusion techniques have less than five LMS patients, effectively precluding meaningful analysis of this subset. In contrast, the CE-MARC study had a LMS population of sufficient size to allow reasonable conclusions to be drawn [13]. SPECT studies of LMS disease have largely been un-blinded,

Table 3 Quantitative CMR perfusion analysis

\begin{tabular}{llll}
\hline & LMS & Control & $P$-value \\
\hline Global stress MBF & $1.77 \pm 0.72$ & $3.28 \pm 1.20$ & $<0.001$ \\
Global rest MBF & $1.28 \pm 0.42$ & $1.48 \pm 0.55$ & 0.14 \\
Global MPR & $1.42 \pm 0.44$ & $2.31 \pm 0.76$ & $<0.001$ \\
LMS territory stress MBF & $2.03 \pm 0.77$ & $3.38 \pm 1.15$ & $<0.001$ \\
LMS territory rest MBF & $1.42 \pm 0.36$ & $1.54 \pm 0.56$ & 0.36 \\
LMS territory MPR & $1.53 \pm 0.44$ & $2.34 \pm 0.64$ & $<0.001$ \\
\hline
\end{tabular}

$\mathrm{MBF}$ values are in $\mathrm{ml} / \mathrm{g} / \mathrm{min}$

$M B F$ myocardial blood flow, MPR myocardial perfusion reserve retrospective and derived from angiographic databases [9-11]; in this context referral bias potentially leads to an over-estimation of the sensitivity of SPECT for the detection of LMS disease [10], as the false negative SPECT scans go unevaluated.

Non-invasive detection of CAD is clinically useful to both determine the presence of clinically significant disease and to estimate the severity and extent of disease. The classical finding of an inducible perfusion abnormality involving both the LAD and LCx coronary artery territories was not robustly seen in LMS patients by either CMR or SPECT. This perfusion defect pattern has been described with varying frequency from 12 to $59 \%$ of SPECT patients with documented significant LMS stenosis in retrospective analyses $[9,11,12]$. This perfusion defect pattern was seen in just $8 \mathrm{LMS}$ patients (30\%) by CMR and 2 patients (7\%) by SPECT in our study. The low diagnostic yield specific for LMS disease may be due, in part, to distal and bifurcation LMS lesions, which may have a differential effect on myocardial perfusion to the LAD and LCx territories, resulting in underestimation of LMS disease. Furthermore, although a visual or QCA reported stenosis of $50 \%$ of the LMS is deemed significant by convention, not all $50 \%$ coronary stenoses are haemodynamically significant when assessed by invasive fractional flow reserve (FFR) [25]. In addition, a myocardial perfusion abnormality consistent with LMS disease may be less apparent in the presence of coronary collateralisation, or flow-limiting stenosis in the right coronary artery (i.e. 3-vessel disease). However, these haemodynamic factors do not account for the differential detection rates of CMR and SPECT (overall $81 \%$ vs. $59 \%$ for detection of CAD). The phenomenon of "balanced ischaemia" in multivessel disease potentially leads to an underestimation of disease, in SPECT this effect is reported 


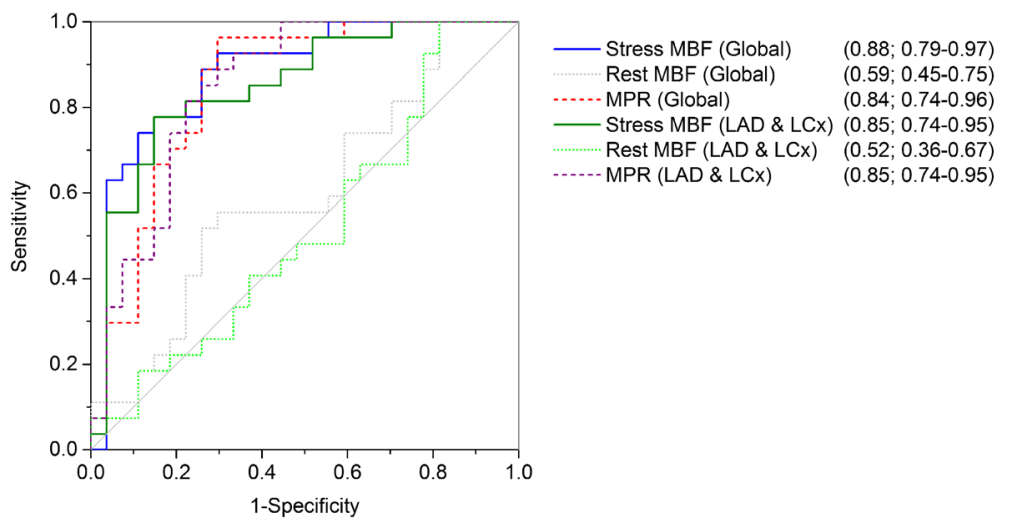

Fig. 3 ROC curves for CMR quantitative perfusion results. Numbers in parentheses indicates AUC with 95\% confidence intervals

with variable frequency [26, 27]. In this context, CMR has been shown to have an advantage over SPECT to detect perfusion defects (in multivessel disease) due to a higher spatial resolution $[28,29]$. Furthermore, multi-vessel disease has been shown to not be significantly associated with false negatives in CMR [30].

Wide interobserver variability for visual severity of stenoses of the LMS have been reported [31, 32]. In our study QCA was used to determine the severity of angiographic stenoses, as per the CE-MARC study protocol $[13,14]$. In this context, there is a potential limitation of the invasive reference standard; however FFR and intravascular ultrasound are only recommended as adjuncts in LMS disease assessment in current guidelines and revascularisation decisions are, for the mainstay, based on severity of angiographic stenosis [33, 34].

Additional diagnostic aids have been proposed to improve the sensitivity of SPECT for the diagnosis of LMS disease. TID of the left ventricular cavity in response to stress has been identified to be a strong predictor of cardiac events [35], reflecting global subendocardial ischaemia or stress-induced left ventricular dysfunction from left main or three vessel disease [36]. Increased right ventricular radiotracer uptake has also been independently associated with LMS disease, with a $60 \%$ increase from $0.33 \pm 0.07$ at rest to $0.51 \pm 0.07$ with stress in LMS patients $(p<0.001$ compared to controls) [37, 38]. When non-perfusion markers of widespread ischaemia are used alongside perfusion data, the proportion of patients with LMS stenosis identified by SPECT increased from 56 to $83 \%$ in one study [9]. In our population, however TID was seen less frequently, with no significant difference in right ventricular uptake between LMS patients and controls suggesting limited discriminatory value. These markers were used for SPECT analysis in this study, but to date have not been used as standard in CMR, and were not prospectively evaluated here.

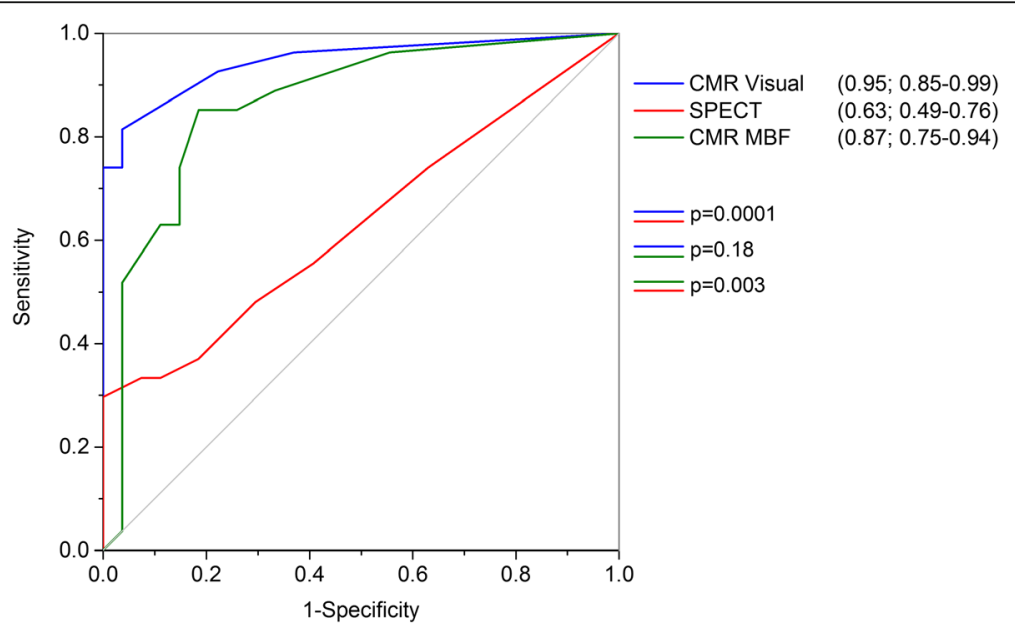

Fig. 4 ROC curves for quantitative summed stress score for MBF, visual CMR and SPECT. Numbers in parentheses indicates AUC with 95\% confidence intervals 
Table 4 Sensitivity, specificity and predictive values for detection of IHD in LMS patients by visual CMR analysis, SPECT and quantitative CMR

\begin{tabular}{lllll}
\hline & Sensitivity & Specificity & PPV* $^{*}$ & NPV* $^{*}$ \\
\hline CMR Visual & 81 & 96 & 48 & 99 \\
CMR MBF & 78 & 85 & 18 & 98 \\
SPECT & 63 & 89 & 19 & 98 \\
\hline
\end{tabular}

Predictive values are corrected based on the prevalence of LMS disease in the CE-MARC population. *PPV positive predictive value, NPV negative predictive value

This study also examined the utility of quantitative CMR perfusion as a potential approach to account for balanced myocardial hypoperfusion that theoretically limits visual analysis in LMS or 3-vessel disease. Other studies have shown that quantitative estimation of myocardial perfusion reserve by CMR over visual analysis improved sensitivity from 74 to $88 \%$ and specificity from 58 to $90 \%$ for patients suspected to have coronary artery disease, but not confined to LMS [39]. The Fermi deconvolution method used in our study has been shown to perform as well as any other model for the detection of CAD [16]. Patel et al. identified increased ischaemia burden by quantitative perfusion methods using Fermi deconvolution over qualitative assessment as severity of coronary disease increased in patients undergoing perfusion CMR with multi-vessel disease [40]. The value of quantitative CMR analysis for LMS lesions has not been previously detailed. In our study, global MBF was the best quantitative marker and showed high sensitivity and specificity (78 and $85 \%$ respectively) for the diagnosis of LMS disease. Quantitative perfusion analysis however was not significantly better than visual CMR perfusion analysis, suggesting that visual perfusion analysis is sufficient to detect heterogeneities in myocardial contrast distribution in LMS disease, a finding supported by dedicated analysis of false-negative CMR [30]. Furthermore, our results suggest there is little additive value to be gained from the quantification of rest perfusion when quantitation of stress perfusion is performed.

\section{Limitations}

Given the low prevalence of LMS disease, the numbers in this prospective study are limited. In our study SPECT analysis did not use attenuation correction; however this was not routine practice when the study was performed [41]. We did not use FFR as our invasive reference standard, however we did use QCA in line with the main CEMARC paper. The pulse sequence used for perfusion imaging in CE-MARC was not fully optimised for quantitative analysis as it used a single preparation pulse for all three slices and a relatively high contrast agent dose. This may have led to a lower performance of quantitative analysis in this study compared to recent approaches. The lack of a completely linear arterial input function measurement for MBF analysis, with the assumption that concentration is linearly related to signal intensity will result in an overestimate of absolute myocardial blood flow. However, post-hoc correction based on baseline signal intensity values would introduce noise into the measurements that could reduce diagnostic accuracy [42]. Furthermore, studies comparing dual-bolus and uncorrected single bolus myocardial blood flow estimates have not shown significant differences in diagnostic accuracy [43]. Our diagnostic accuracy values agree well with other studies in the literature, suggesting that these limitations have not significantly impacted on our findings.

\section{Conclusion}

This study shows that visual stress perfusion CMR had higher diagnostic accuracy than SPECT to detect significant LMS or LMS equivalent disease. Quantitative perfusion CMR by Fermi-constrained deconvolution had similar performance to visual CMR perfusion analysis.

\section{Abbreviations \\ AUC: Area under the curve; CAD: Coronary artery disease; CE-MARC: Clinical Evaluation of MAgnetic Resonance imaging in Coronary heart disease; CMR: Cardiovascular magnetic resonance; FFR: Fractional flow reserve; LAD: Left anterior descending coronary artery; LCx: left circumflex coronary artery; LGE: late gadolinium enhancement; LMS: Left main stem; LV: Left ventricle/ left ventricular; MBF: Myocardial blood flow; MPR: Myocardial perfusion reserve; QCA: Quantitative coronary angiography; ROC: Receiver operating characteristic; SPECT: Single-photon emission computed tomography; SSS: Summed stress score; TID: Transient ischaemic dilatation}

\section{Acknowledgements}

This study was funded by the British Heart Foundation. We thank for their assistance Gavin Bainbridge and Margaret Saysell (cardiac radiographers); Petra Bijsterveld, Fiona Richards and Judith Beevers (CE-MARC clinical research nurses); Penelope Thorley (clinical scientist in nuclear medicine); and Peter Tooze (database design and maintenance).

\section{Funding}

CE-MARC was funded by the British Heart Foundation (BHF), grant reference RG/05/004.

Availability of data and materials

All data generated or analysed during this pre-specified sub-study are included in this published article.

\section{Disclosures}

JPG and SP have received an educational research grant from Philips Healthcare. The remaining authors have nothing to disclose.

\section{Authors' contributions \\ JF collected data, analysed the CMR perfusion data and drafted the manuscript, AK assisted with statistical analysis, JDB analysed the quantitative CMR perfusion data, NM helped with collecting data and CD analysed the SPECT data, SP and JPG analysed CMR data and conceived the trial design. All authors read and approved the final manuscript.}

\section{Ethics approval and consent to participate}

The study was performed in accordance with the Declaration of Helsinki (October 2000), with all patients providing informed written consent. The study protocol and other relevant documentation had been approved by the National Research Ethics Committee. 


\section{Competing interests}

JPG and SP have received an educational research grant from Philips Healthcare. The remaining authors declare they have no competing interests.

\section{Publisher's Note}

Springer Nature remains neutral with regard to jurisdictional claims in published maps and institutional affiliations.

\begin{abstract}
Author details
${ }^{1}$ Multidisciplinary Cardiovascular Research Centre \& The Division of Biomedical Imaging, Leeds Institute of Cardiovascular and Metabolic Medicine, University of Leeds, Leeds LS2 9JT, UK. ${ }^{2}$ Cardiology Department, Leeds General Infirmary, Leeds, UK.
\end{abstract}

Received: 9 June 2017 Accepted: 16 October 2017

\section{Published online: 06 November 2017}

\section{References}

1. El-Menyar AA, Al Suwaidi J, Holmes DR. Left main coronary artery stenosis: state-of-the-art. Curr Probl Cardiol. 2007;32:103-93.

2. Conley MJ, Ely RL, Kisslo J, Lee KL, McNeer JF, Rosati RA. The prognostic spectrum of left main stenosis. Circulation. 1978;57:947-52.

3. Taylor H a, Deumite NJ, Chaitman BR, Davis KB, Killip T, Rogers WJ. Asymptomatic left main coronary artery disease in the coronary artery surgery study (CASS) registry. Circulation. 1989;79:1171-9.

4. Serruys PW, Morice M-C, Kappetein AP, Colombo A, Holmes DR, Mack MJ, et al. Percutaneous coronary intervention versus coronary-artery bypass grafting for severe coronary artery disease. N Engl J Med. 2009;360:961-72.

5. Naik H, White AJ, Chakravarty T, Forrester J, Fontana G, Kar S, et al. A metaanalysis of 3,773 patients treated with percutaneous coronary intervention or surgery for unprotected left main coronary artery stenosis. JACC Cardiovasc Interv. 2009;2:739-47.

6. Beller GA, Zaret BL. Contributions of nuclear cardiology to diagnosis and prognosis of patients with coronary artery disease. Circulation. 2000;101:1465-78.

7. Lipinski MJ, McVey CM, Berger JS, Kramer CM, Salerno M. Prognostic value of stress cardiac magnetic resonance imaging in patients with known or suspected coronary artery disease: a systematic review and meta-analysis. J Am Coll Cardiol. 2013:62:826-38.

8. Greenwood JP, Herzog BA, Brown JM, Everett CC, Nixon J, Bijsterveld P, et al. Prognostic value of cardiovascular magnetic resonance and singlephoton emission computed tomography in suspected coronary heart disease: long-term follow-up of a prospective, diagnostic accuracy cohort study. Ann Intern Med. 2016:165:1-10.

9. Berman DS, Kang X, Slomka PJ, Gerlach J, de Yang L, Hayes SW, et al. Underestimation of extent of ischemia by gated SPECT myocardial perfusion imaging in patients with left main coronary artery disease. J Nucl Cardiol. 2007;14:521-8.

10. Afonso L, Mahajan N. Single-photon emission computed tomography myocardial perfusion imaging in the diagnosis of left main disease. Clin Cardiol. 2009;32:E11-5.

11. Shiba C, Chikamori T, Hida S, Igarashi Y, Tanaka H, Hirose K-I, et al. Important parameters in the detection of left main trunk disease using stress myocardial perfusion imaging. J Cardiol. 2009;53:43-52.

12. Rehn T, Griffith LS, Achuff SC, Bailey IK, Bulkley BH, Burow R, et al. Exercise thallium-201 myocardial imaging in left main coronary artery disease: sensitive but not specific. Am J Cardiol. 1981;48:217-23.

13. Greenwood JP, Maredia N, Younger JF, Brown JM, Nixon J, Everett CC, et al. Cardiovascular magnetic resonance and single-photon emission computed tomography for diagnosis of coronary heart disease (CE-MARC): a prospective trial. Lancet. 2012;379:453-60.

14. Greenwood JP, Maredia N, Radjenovic A, Brown JM, Nixon J, Farrin AJ, et al. Clinical evaluation of magnetic resonance imaging in coronary heart disease: the CE-MARC study. Trials. 2009;10:62.

15. Cerqueira MD, Weissman NJ, Dilsizian V, Jacobs AK, Kaul S, Laskey WK, et al. Standardized myocardial segmentation and nomenclature for tomographic imaging of the heart. A statement for healthcare professionals from the cardiac imaging Committee of the Council on clinical cardiology of the American Heart Association. Circulation. 2002;105:539-42.

16. Biglands JD, Magee DR, Sourbron SP, Plein S, Greenwood JP, Radjenovic A. Comparison of the diagnostic performance of four quantitative myocardial perfusion estimation methods used in cardiac MR imaging: CE-MARC substudy. Radiology. 2015;275:393-402.

17. Jerosch-Herold M, Wilke N, Stillman AE. Magnetic resonance quantification of the myocardial perfusion reserve with a Fermi function model for constrained deconvolution. Am Assoc Phys Med. 1998;25:73-84.

18. Biglands J, Magee D, Boyle R, Larghat A, Plein S, Radjenović A. Evaluation of the effect of myocardial segmentation errors on myocardial blood flow estimates from DCE-MRI. Phys Med Biol. 2011;56:2423-43.

19. DeLong ER, DeLong DM, Clarke-Pearson DL. Comparing the areas under two or more correlated receiver operating characteristic curves: a nonparametric approach. Biometrics. 1988;44:837-45.

20. Youden WJ. Index for rating diagnostic tests. Cancer. 1950;3:32-5.

21. Greenwood JP, Motwani M, Maredia N, Brown JM, Everett CC, Nixon J, et al. Comparison of cardiovascular magnetic resonance and single-photon emission computed tomography in women with suspected coronary artery disease from the clinical evaluation of magnetic resonance imaging in coronary heart disease (CE-MARC) trial. Circulation. 2014;129:1129-38.

22. Schwitter J, Wacker CM, Wilke N, Al-Saadi N, Sauer E, Huettle K, et al. MRIMPACT II: magnetic resonance imaging for myocardial perfusion assessment in coronary artery disease trial: perfusion-cardiac magnetic resonance vs. single-photon emission computed tomography for the detection of coronary artery disease: a comparative. Eur. Heart J. 2013;34:775-81.

23. Walker S, Girardin F, McKenna C, Ball SG, Nixon J, Plein S, et al. Costeffectiveness of cardiovascular magnetic resonance in the diagnosis of coronary heart disease: an economic evaluation using data from the CEMARC study. Heart. 2013;99:873-81.

24. Schwitter J, Wacker CM, Van Rossum AC, Lombardi M, Al-Saadi N, Ahlstrom $H$, et al. MR-IMPACT: comparison of perfusion-cardiac magnetic resonance with single-photon emission computed tomography for the detection of coronary artery disease in a multicentre, multivendor, randomized trial. Eur Heart J 2008;29:480-489.

25. Pijls NHJ, De Bruyne B, Peels K, Van Der Voort PH, Bonnier HJRM, Bartunek J, Koolen JJ, et al. Measurement of fractional flow reserve to assess the functional severity of coronary-artery stenoses. N Engl J Med. 1996;334:1703-8.

26. Reyes E. Detection of left main stem and three-vessel coronary artery disease by myocardial perfusion SPECT imaging. Eurolntervention. 2010;6:G72-8.

27. Ragosta M, Bishop AH, Lipson LC, Watson DD, Gimple LW, Sarembock IJ, et al. Comparison between angiography and fractional flow reserve versus single-photon emission computed Tomographic myocardial perfusion imaging for determining lesion significance in patients with multivessel coronary disease. Am J Cardiol. 2007;99:896-902.

28. Chung S-Y, Lee K-Y, Chun EJ, Lee W-W, Park EK, Chang H-J, et al. Comparison of stress perfusion MRI and SPECT for detection of myocardial ischemia in patients with angiographically proven three-vessel coronary artery disease. AJR Am J Roentgenol. 2010;195:356-62.

29. Schwitter J, Wacker CM, Wilke N, Al-Saadi N, Sauer E, Huettle K, et al. Superior diagnostic performance of perfusion-cardiovascular magnetic resonance versus SPECT to detect coronary artery disease: the secondary endpoints of the multicenter multivendor MR-IMPACT I| (magnetic resonance imaging for myocardial perfusion Assessment in Coronary Artery Disease Trial). J Cardiovasc Magn Reson. 2012;14:61.

30. Kidambi A, Sourbron S, Maredia N, Motwani M, Brown JM, Nixon J, et al. Factors associated with false-negative cardiovascular magnetic resonance perfusion studies: a clinical evaluation of magnetic resonance imaging in coronary artery disease (CE-MARC) substudy. J Magn Reson Imaging. 2016;43:566-73.

31. Isner JM, Kishel J, Kent KM, Ronan JA, Ross AM, Roberts WC. Accuracy of angiographic determination of left main coronary arterial narrowing. Angiographichistologic correlative analysis in 28 patients. Circulation. 1981;63:1056-64.

32. Cameron A, Kemp HG, Fisher LD, Gosselin A, Judkins MP, Kennedy JW, et al. Left main coronary artery stenosis: angiographic determination. Circulation. 1983;68:484-9.

33. Fihn SD, Gardin JM, Abrams J, Berra K, Blankenship JC, Dallas AP, et al. 2012 ACCF/AHA/ACP/AATS/PCNA/SCAI/STS guideline for the diagnosis and management of patients with stable ischemic heart disease: a report of the American College of Cardiology Foundation/American Heart Association task force on practice guidelines, and the. J Am Coll Cardiol. 2012;60:e44-164.

34. Windecker S, Kolh P, Alfonso F, Collet J-P, Cremer J, Falk V, et al. 2014 ESC/ EACTS guidelines on myocardial revascularization. Eurolntervention. 2015;10:1024-94.

35. Abidov A, Bax JJ, Hayes SW, Hachamovitch R, Cohen I, Gerlach J, et al. Transient ischemic dilation ratio of the left ventricle is a significant predictor 
of future cardiac events in patients with otherwise normal myocardial perfusion SPECT. J Am Coll Cardiol. 2003;42:1818-25.

36. McLaughlin MG, Danias PG. Transient ischemic dilation: a powerful diagnostic and prognostic finding of stress myocardial perfusion imaging. J Nucl Cardiol. 2002;9:663-7.

37. Williams KA, Schneider CM. Increased stress right ventricular activity on dual isotope perfusion SPECT: a sign of multivessel and/or left main coronary artery disease. J Am Coll Cardiol. 1999;34:420-7.

38. Mannting F, Zabrodina W, Dass C. Significance of increased right ventricular uptake on $99 \mathrm{mTc}$-sestamibi SPECT in patients with coronary artery disease. J Nucl Med. 1999;40:889-94.

39. Nagel E, Klein C, Paetsch I, Hettwer S, Schnackenburg B, Wegscheider K, et al. Magnetic resonance perfusion measurements for the noninvasive detection of coronary artery disease. Circulation. 2003;108:432-7.

40. Patel AR, Antkowiak PF, Nandalur KR, West AM, Salerno M, Arora V, et al. Assessment of advanced coronary artery disease: advantages of quantitative cardiac magnetic resonance perfusion analysis. J Am Coll Cardiol. 2010;56: $561-9$.

41. Greenwood JP, Brown JM, Dickinson CJ, Ball SG, Plein S. CMR versus SPECT for diagnosis of coronary heart disease - authors' reply. Lancet. 2012;379: 2147-8.

42. Broadbent DA, Biglands JD, Ripley DP, Higgins DM, Greenwood JP, Plein S, et al. Sensitivity of quantitative myocardial dynamic contrast-enhanced MRI to saturation pulse efficiency, noise and t1 measurement error: comparison of nonlinearity correction methods. Magn Reson Med. 2016;75:1290-300.

43. Groothuis JGJ, Kremers FPPJ, Beek AM, Brinckman SL, Tuinenburg AC, Jerosch-Herold M, et al. Comparison of dual to single contrast bolus magnetic resonance myocardial perfusion imaging for detection of significant coronary artery disease. J Magn Reson Imaging. 2010;32:88-93.

\section{Submit your next manuscript to BioMed Central and we will help you at every step:}

- We accept pre-submission inquiries

- Our selector tool helps you to find the most relevant journal

- We provide round the clock customer support

- Convenient online submission

- Thorough peer review

- Inclusion in PubMed and all major indexing services

- Maximum visibility for your research

Submit your manuscript at www.biomedcentral.com/submit
C Biomed Central 